\title{
Technical note: Comparison of radial immunodiffusion and ELISA for quantification of bovine immunoglobulin $\mathbf{G}$ in colostrum and plasma
}

\author{
S. L. Gelsinger, A. M. Smith, C. M. Jones, and A. J. Heinrichs ${ }^{1}$ \\ Department of Animal Science, Pennsylvania State University, University Park 16802
}

\section{ABSTRACT}

Historically, radial immunodiffusion (RID) has been the only method that directly measures IgG; however, recent studies have reported IgG concentrations in colostrum, milk, and plasma as measured using an ELISA. To our knowledge no comparison between RID and ELISA methods has been made for bovine colostrum or plasma. The objective of this study was to compare IgG concentrations measured by both methods in samples of bovine colostrum before and after heat treatment and bovine plasma. Concentration of $\operatorname{IgG}$ was quantified using a commercially available RID kit and a modified ELISA. Samples of bovine colostrum and plasma were collected from individual animals and colostrum was tested before and after heat treatment at $60^{\circ} \mathrm{C}$ for 30 min. All samples were tested using both methods. Pearson correlation coefficients were determined for RID and ELISA values from unheated colostrum, heattreated colostrum, and plasma samples. Mixed models were used to determine the effect of assay on IgG measurement in colostrum and plasma and effect of heat treatment on IgG concentration in colostrum. A weak correlation was found between ELISA and RID results in plasma and unheated colostrum. Concentration of IgG was significantly lower in all sample types when measured by ELISA compared to RID. Thus, direct comparison of ELISA and RID results is not recommended. Colostrum IgG concentration significantly decreased after heat treatment as measured by ELISA, but means were not different when measured by RID. Correlation plots between colostrum values measured before and after heat treatment indicated changes in the colostrum protein matrix due to heat affected RID and ELISA assays differently. This investigation compared RID and ELISA results, but no conclusions could be drawn as to the accuracy of either assay.

Key words: radial immunodiffusion, ELISA, IgG, calf, colostrum

Received June 16, 2014.

Accepted February 19, 2015.

${ }^{1}$ Corresponding author: ajh@psu.edu

\section{Technical Note}

Absorption from colostrum is the only method by which bovine neonates receive the immunoglobulins that defend against disease in the first weeks of life. The most prevalent immunoglobulin in bovine colostrum is IgG (Butler, 1969). Concentration of IgG is routinely measured in colostrum and in the blood of calves 24 to $48 \mathrm{~h}$ after birth to determine colostrum quality and assess success or failure of passive transfer of immunity. To ensure that calves receive an adequate mass of IgG, at least $4 \mathrm{~L}$ of colostrum containing at least $50 \mathrm{~g} / \mathrm{L}$ of IgG should be fed as soon as possible after birth (Godden, 2008). A radial immunodiffusion (RID) method has been developed and successfully used for determining IgG concentration in milk, colostrum, and blood plasma; RID results have been used in the development of other methods correlating IgG concentration with specific gravity, refraction index, or plasma total protein to estimate IgG concentration (Pritchett et al., 1994, Tyler et al., 1996; Weaver et al., 2000; Bielmann et al., 2010). Historically, RID has been the only method that directly measures IgG; however, recent studies have reported IgG concentrations in colostrum, milk, and plasma as measured using an ELISA (Vetter et al., 2013; Baumrucker and Bruckmaier, 2014; Baumrucker et al., 2014; Gelsinger et al., 2015). To our knowledge no comparison between RID and ELISA methods has been made for bovine colostrum or plasma. Considerable cost may be accrued by routine performance of RID due to limited commercial availability. An ELISA may be an economical alternative method.

Heat treatment of colostrum is a method developed to reduce calf exposure to pathogens. Several studies have reported no effect of heat treatment on colostrum IgG concentration (Godden et al., 2006; ElizondoSalazar et al., 2010). However, these studies used RID for measuring colostrum IgG concentration. The effect of heat treatment has not been assessed using ELISA, which may be a more sensitive method.

The objective of the present experiment was to measure IgG concentration in colostrum samples before and after heat treatment and also in plasma using both RID and ELISA methods and to compare results from 
each method. We hypothesized that results from each method would be positively correlated and that the ELISA procedure would give more precise estimates of IgG concentration.

Colostrum samples $(\mathrm{n}=58)$ were collected from individual cows at the first milking after calving and frozen until needed for analysis. Blood samples were obtained $48 \mathrm{~h}$ after birth from 104 bull calves that were fed either unheated or heat-treated colostrum. Each calf received $4 \mathrm{~L}$ of colostrum within $8 \mathrm{~h}$ after birth. Blood samples were centrifuged $\left(1,500 \times g\right.$ at $\left.4^{\circ} \mathrm{C}\right)$ and plasma was collected and frozen until needed for analysis.

The RID procedure was performed according to manufacturer instructions (\#728411; Triple J Farms, Bellingham, WA). One RID plate with anti-bovine IgG antibody within an agarose gel (0.1 $M$ phosphate buffer, $0.1 \%$ sodium azide, $1 \mathrm{ug} / \mathrm{mL}$ of amphotericin B, $0.002 M$ EDTA; pH 7.0) and 3 standard solutions of bovine serum $(1.96,14.02$, and $27.48 \mathrm{~g}$ of $\mathrm{IgG} / \mathrm{L}$ ) were included in each kit. Standard solutions were used as provided, without dilution, for the plate with which they originated. A plasma sample of known IgG concentration was included in each assay as a positive control.

To ensure that colostrum IgG concentration fell within the range of the standards provided, samples were diluted $1: 10$ in a $0.85 \%$ saline solution. The full process was duplicated for each sample. Precipitin ring diameters were measured after a 24 -h incubation at $20^{\circ} \mathrm{C}$ using a peak scale loupe $7 \times$ (\#1975; GWJ Company, Peak Optics, Hacienda Heights, CA). Mean IgG concentration and standard deviation were calculated from results of duplicated tests and used to calculate a coefficient of variation for each sample. Inter and intra-assay coefficients of variation were $\leq 10.5 \%$. Samples with coefficient of variation $>10.5 \%$ were retested. Plasma samples were tested in duplicate wells but without dilution as plasma IgG concentrations were expected to be within the range of the provided standards.

The ELISA procedure used for colostrum and plasma was adapted from instructions provided by the manufacturer (Bethyl Laboratories, Montgomery, TX) and the protocol described by Vetter et al. (2013). Purified IgG (1 mg IgG/mL; \#P10-115; Bethyl Laboratories) was diluted using Tris buffer saline with Tween 20 (TBS-Tween; $50 \mathrm{~m} M$ Tris, $0.14 M$ sodium chloride, $0.05 \%$ Tween $20, \mathrm{pH} 8.0$ ) to create 7 standard solutions $(1,000,500,250,125,62.5,31.25$, and $15.125 \mathrm{ng}$ of $\mathrm{IgG} / \mathrm{mL})$. Standard solutions were made in test tubes, thoroughly mixed by vortexing individual tubes, and transferred into wells at the appropriate time in the ELISA protocol. Duplicate standard dilution series were created for each assay and TBS-Tween served as a negative control.
Whole colostrum was diluted using TBS-Tween with final dilution factors of $1 \times 10^{6}, 2 \times 10^{6}, 4 \times 10^{6}$, and $8 \times 10^{6}$. Dilution series were created in separate test tubes and final solutions were transferred to individual wells at the appropriate time in the ELISA protocol. All dilutions were duplicated so that a total of 8 wells ( 2 wells per dilution factor) contained solutions representing the same colostrum sample. Wells were coated with $100 \mu \mathrm{L}$ of affinity-purified $\mathrm{IgG}(2 \mu \mathrm{g} / \mathrm{mL}$; \#A10 118A; Bethyl Laboratories). A $0.05 \%$ solution of gelatin from cold water fish skin (\#G7041; Sigma-Aldrich Inc., St. Louis, MO) was used as a blocking agent and 150 $\mu \mathrm{L}$ were added to each well. Horseradish peroxidase conjugated antibody produced in sheep against bovine IgG heavy chain (A10-118P; Bethyl Laboratories) was used to detect IgG concentration in standard and sample solutions. A working solution containing $5 \mathrm{ng} / \mathrm{mL}$ of detection antibody was created using TBS-Tween. Each well received $100 \mu \mathrm{L}$ of the detection antibody working solution. The enzyme reaction was carried out using $100 \mu \mathrm{L}$ of $3,3^{\prime}, 5,5^{\prime}$-tetramethylbenzidine ELISA peroxidase substrate (Rockland Immunochemicals Inc., Gilbertsville, PA) and terminated with $100 \mu \mathrm{L}$ of 0.2 $M$ sulfuric acid. Individual plates were incubated at $20^{\circ} \mathrm{C}$ for $60 \mathrm{~min}$ after each step and wells were washed 5 times with TBS-Tween before adding the reagent for the subsequent step. The only exception occurred in the enzyme reaction and addition of $0.2 \mathrm{M}$ sulfuric acid; the enzyme reaction was allowed to proceed for 10 to 30 min and wells were not washed before adding sulfuric acid.

Plasma was diluted in TBS-Tween to a final dilution factor of $5 \times 10^{5}$. All dilutions were duplicated and tested in 4 separate wells such that a total of 8 wells contained solutions representing the same plasma sample. Reagents and protocols are described above, except that standard solutions ranged from 3.125 to $200 \mathrm{ng}$ of $\mathrm{IgG} / \mathrm{mL}$. This narrower range of standards and a single final dilution factor $(50,000 \times)$ were used for plasma because plasma IgG concentration was expected to be less variable than colostrum.

Absorbance was read using a plate reader $\left(\right.$ Victor $^{3}$ Multilabel Counter model 1420, PerkinElmer Life Sciences, Waltham, MA) at a wavelength of $450 \mathrm{~nm}$. Absorbance values from wells containing negative controls were averaged and subtracted from standard and sample absorbance values. Duplicate standard values were averaged and used to create a standard curve relating absorbance to IgG concentration. Results from individual assays were used only when the coefficient of determination value from the standard curve exceeded 0.9. Results from assays with unacceptable coefficient of determination values were disregarded, and the samples retested in subsequent assays. Mean IgG concentration 
and standard deviation were calculated for each sample and used to determine coefficient of variation. Values were accepted when the coefficient of variation from at least 3 of 4 wells representing a single dilution series was $10.5 \%$ or less and the coefficient of variation from both dilution series for the same sample was $\leq 15 \%$. Samples with a coefficient of variation greater than these criteria were retested and final dilution factors were adjusted accordingly.

When final values with a coefficient of variation $\leq 10.5 \%$ were obtained from each assay for each colostrum sample, aliquots of each sample were heat treated by raising and maintaining the colostrum temperature at $60^{\circ} \mathrm{C}$ for 30 min using a water bath and subsequently cooling on ice (Elizondo-Salazar et al., 2010). Water temperature and temperature of several colostrum samples were monitored continuously during heat treatment. The IgG concentration of each sample was reevaluated after heat treatment using the same procedures and acceptance criteria described previously.

Results from ELISA were regressed on RID results using PROC REG in SAS (Version 9.3, SAS Institute Inc., Cary, NC) to obtain studentized residuals and leverage values for each sample. Samples with studentized residuals $>2$ or leverage values $>0.04$ for plasma or $>0.07$ for colostrum were retested in both assays to confirm accuracy of results. Confirmed data points were entered into the model and measures of influence were reevaluated. Plasma samples whose final leverage values were $>0.04$ and colostrum samples whose final leverage values were $>0.07$ were not used in the final analysis. Eight heat-treated and 6 unheated colostrum samples and 5 plasma samples were removed from final analyses due to high leverage values.

The CORR procedure in SAS was used to find Pearson correlation coefficients for unheated colostrum, heat-treated colostrum, and plasma samples and to compare colostrum values measured before and after heat treatment by each test. Final sample size used to compare colostrum IgG before and after heat treatment for each test was 45 because 1 sample was an outlier in both the heated and unheated data sets. Normality and constant variance assumptions were assessed using diagnostic plots and Levene's homogeneity of variance test in PROC GLM. Plasma and colostrum data were both normally distributed and exhibited constant variance across all IgG concentrations. The MIXED procedure was used to compare IgG concentrations between the 2 assays for colostrum and plasma and in colostrum before and after heat treatment. Assay type (RID, ELISA), treatment (unheated, heat-treated) and their interaction were entered as fixed effects in the model for colostrum IgG concentration. Assay type
(RID, ELISA) was the only fixed effect in the model for plasma IgG concentration. Colostrum or plasma sample was included as a random effect in the respective model. Denominator degrees of freedom were calculated using the Kenward-Rogers method. Least squares means were determined and compared using the Tukey adjustment for multiple comparisons.

Mean $( \pm \mathrm{SD})$ coefficient of determination values for the standard curves from individual ELISA and RID assays were $0.96( \pm 0.03)$ and $0.99( \pm 0.02)$, respectively. Scatter plots and Pearson coefficients for unheated colostrum, heat-treated colostrum, and plasma are shown in Figure 1. Results from ELISA were most closely correlated to RID results in plasma samples $(\mathrm{r}=0.59, P$ $<0.01$ ). However, this is a relatively weak correlation. The relationship was weaker in unheated colostrum but still statistically significant. Results from ELISA and RID were not significantly correlated in colostrum after heat treatment.

Results from the mixed model analysis are shown in Table 1. Assay type significantly affected IgG concentration in both plasma and colostrum. In both cases, ELISA values were consistently lower than RID. Values from ELISA measurement may be similar to IgG concentrations measured by turbidimetric immunoassay, which also reports IgG concentrations consistently lower than RID (Quigley et al., 2013). Comparison of ELISA and turbidimetric immunoassay is beyond the scope of the present investigation.

Figure 2 shows the distribution of both heat-treated and unheated colostrum and plasma samples by the number of repeated analyses required to meet preset acceptance criteria. A greater proportion of samples required repeated analysis when tested with ELISA compared with RID. As a reminder, IgG concentration values were not accepted from either method unless they agreed with a coefficient of variation $<10.5 \%$ within a plate and $15 \%$ between plates. Our results indicated that the RID is a more consistent method. The variation in ELISA results may be due to the extensive dilutions required to test plasma and colostrum IgG concentration using this method compared with RID, which requires minimal sample dilution. Within the ELISA, plasma samples required more repetition than either heated or unheated colostrum. Colostrum samples were diluted 10 to 100 fold more than plasma; this has been shown to eliminate the matrix effect of colostrum (Baumrucker et al., 2014). The antibodies used in this ELISA were validated for use in serum and plasma, thus we did not test for potential matrix effects; however, researchers utilizing this method in the future may consider quantifying these effects. The number of times samples were repeated was also influ- 
Table 1. Least squares means and SEM for IgG concentration in plasma and colostrum measured by ELISA or radial immunodiffusion (RID) methods and colostrum before and after heat treatment

\begin{tabular}{lcccccc}
\hline & & & \multicolumn{3}{c}{$P$-value $^{1}$} \\
\cline { 5 - 7 } Item & ELISA & RID & SEM & T & $\mathrm{H}$ & $\mathrm{T} \times \mathrm{H}$ \\
\hline Plasma IgG, $(\mathrm{mg} / \mathrm{mL})$ & $11.2^{\mathrm{b}}$ & $14.7^{\mathrm{a}}$ & 0.8 & 0.01 & - & - \\
Colostrum IgG $(\mathrm{mg} / \mathrm{mL})$ & $40.08^{\mathrm{b}}$ & $77.94^{\mathrm{a}}$ & 2.8 & & & 0.02 \\
Unheated & $20.23^{\mathrm{c}}$ & $69.47^{\mathrm{a}}$ & 2.8 & & & \\
Heat-treated & &
\end{tabular}

${ }^{\mathrm{a}, \mathrm{b}}$ Within plasma or colostrum values with different superscripts are significantly different from each other $(P$ $<0.05)$.

${ }^{1} \mathrm{~T}=$ fixed effect of test, $\mathrm{H}=$ fixed effect of heat treatment.

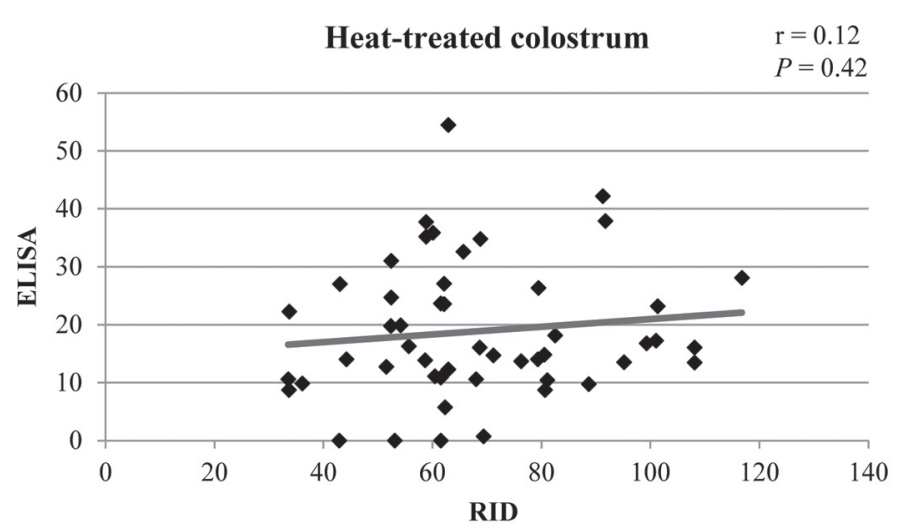

enced by whether the IgG concentration fell within the range of standards used for the assay. Plasma samples were tested using a narrower range of standards to create a more linear curve and reduce coefficient of variation among values for the same sample. However, this resulted in samples falling above the highest standards and requiring retesting using a different dilution factor. Maintaining a larger range of standards is recommended in subsequent research.
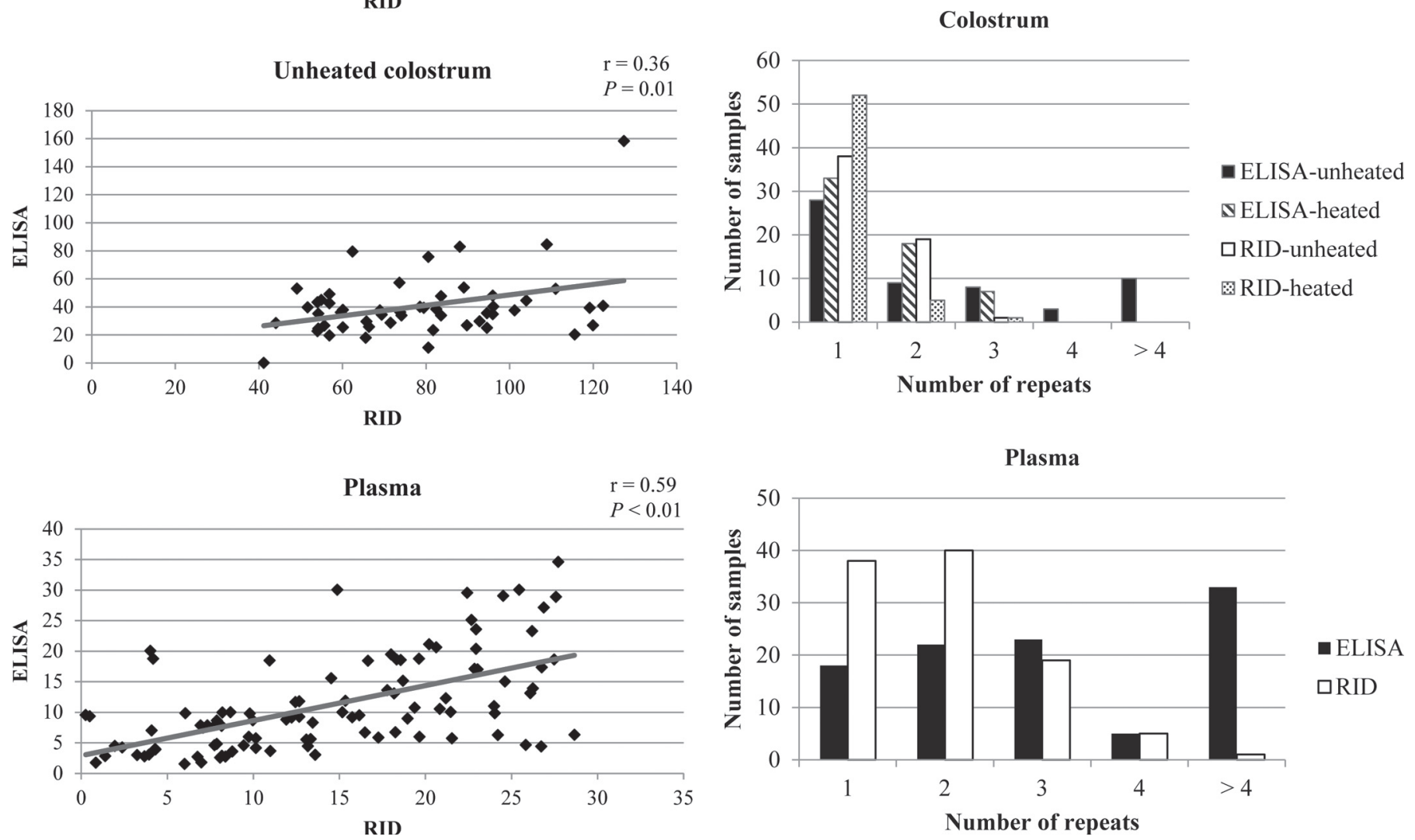

Figure 1. Correlation plots of IgG concentration measured by radial immunodiffusion (RID) and ELISA in heat-treated colostrum, unheated colostrum, and plasma.

Figure 2. Histograms showing the number of repeated analyses by ELISA and radial immunodiffusion (RID) for unheated and heattreated $(\mathrm{n}=58)$ colostrum as well as plasma $(\mathrm{n}=104)$ samples. 

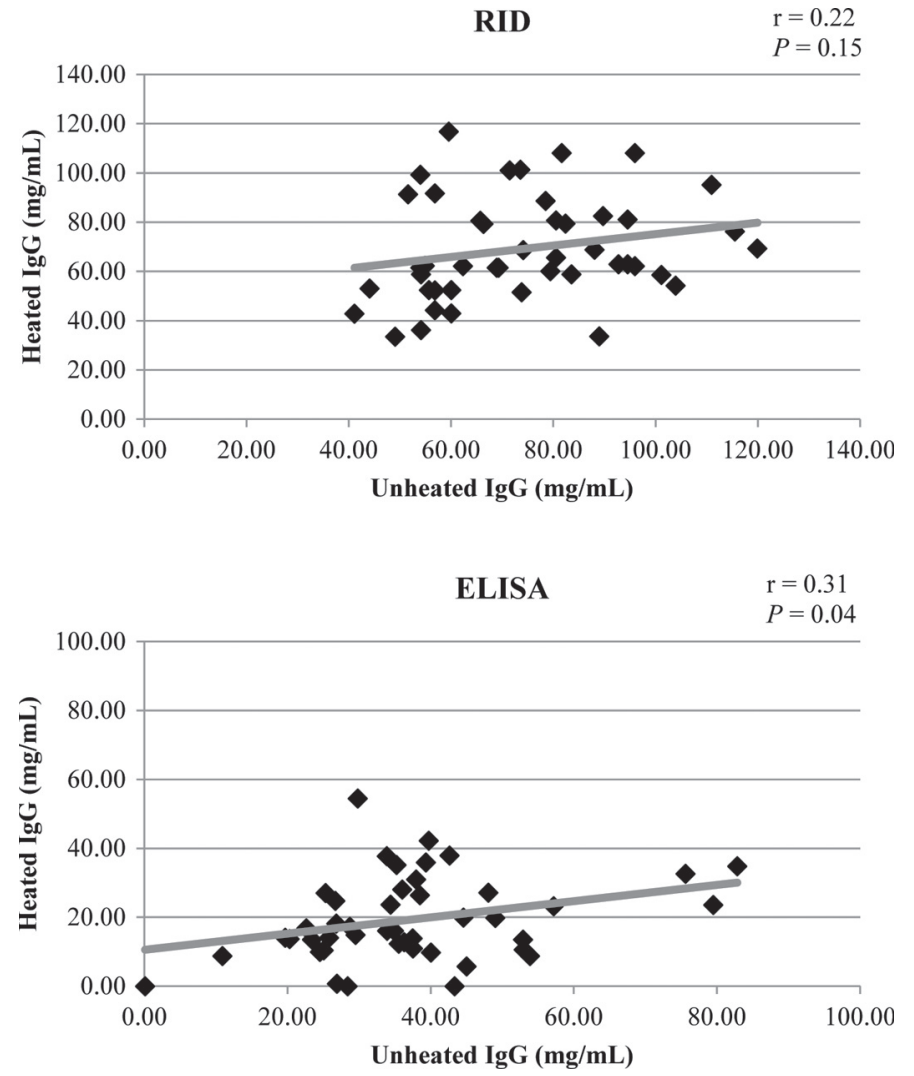

Figure 3. Correlation of IgG concentration measured by radial immunodiffusion (RID) and ELISA methods in colostrum samples $(\mathrm{n}=$ 45) before and after heat-treatment.

Heat treatment reduced IgG concentration as measured by ELISA, but not RID. Correlation plots for IgG concentrations of samples measured before and after heating are shown for both ELISA and RID methods in Figure 3. Values for the same sample measured before and after heating were more closely correlated when measured by ELISA than RID. This is surprising considering that the overall mean $\operatorname{IgG}$ concentration measured by RID did not change after colostrum was heated, and implies that the effects of heat on the colostrum protein matrix affects the ELISA and RID assays differently. The effect of heat treatment on non-IgG colostrum components has not been investigated. Baumrucker et al. (2014) referenced the potential matrix effect on ELISA results and concluded that the matrix effect in raw colostrum is minimized by extensive dilution of colostrum samples. It is unknown how heat may change the matrix and affect ELISA or RID measurements. This may be an explanation for the lack of correlation between tests in heated colostrum.

Previous studies using a serum neutralization assay to investigate the ability of IgG to recognize and bind antigen found no difference in $\operatorname{IgG}$ from unheated or heat-treated colostrum (McMartin et al., 2006; Johnson et al., 2007). Results from these studies are based on a few samples and must be interpreted with caution; however, other studies investigating calf health have reported no difference or improved health in calves that received heat-treated colostrum compared with calves fed unheated colostrum (Elizondo-Salazar and Heinrichs, 2009; Godden et al., 2012). Thus, the decrease in IgG concentration indicated by ELISA may not be of practical significance.

Plasma IgG concentration was also significantly lower when measured with ELISA. Plasma IgG in calves between 24 and $48 \mathrm{~h}$ of age is used as an indicator of passive transfer of immunity. The cutoff value for successful passive transfer (10 $\mathrm{mg}$ of $\mathrm{IgG} / \mathrm{mL}$ ) was established from RID measurements of many serum and plasma samples (Tyler et al., 1996). Results from ELISA were significantly correlated with RID values but, due to the relative weakness of this correlation and consistent underestimation of IgG concentration by ELISA compared with RID, direct comparison of ELISA and RID values is not recommended. Thus, if ELISA is to be routinely used to assess passive transfer in calves, it may be necessary to establish a new cutoff value for determining successful passive transfer using ELISA. Otherwise, calves may be misdiagnosed as experiencing failure of passive transfer.

Similarly, the cutoff value for determining goodquality colostrum, suitable for feeding to calves $(>50$ $\mathrm{mg}$ of $\mathrm{IgG} / \mathrm{mL}$; Godden, 2008), has been determined by measuring many colostrum samples by RID. If ELISA is to be used routinely for assessing colostrum quality, it may be necessary to establish new cutoff values for good-quality colostrum. Due to the extensive laboratory time, reagents, and equipment necessary for the ELISA protocol, it is unlikely that ELISA will be used by dairy producers. However, ELISA may be considered by other investigators as an economical alternative to RID in future colostrum research.

Concentration of IgG measured by ELISA and RID was only weakly correlated in plasma and unheated colostrum, and uncorrelated in heat-treated colostrum. Concentrations measured with ELISA were significantly lower than RID results in colostrum and plasma, and heat treatment of colostrum significantly reduced IgG concentration in colostrum when measured by ELISA. No effect of heat treatment was detected by RID. Based on these results, direct comparison of ELISA and RID results from colostrum or plasma IgG concentration is not recommended. However, it may be possible to draw conclusions from a comparison of IgG concentration among samples measured by the same method. 


\section{REFERENCES}

Baumrucker, C. R., and R. M. Bruckmaier. 2014. Colostrogenesis: IgG(1) transcytosis mechanisms. J. Mammary Gland Biol. Neoplasia 19:103-117.

Baumrucker, C. R., A. Stark, O. Wellnitz, C. Dechow, and R. M. Bruckmaier. 2014. Short communication: Immunoglobulin variation in quarter-milked colostrum. J. Dairy Sci. 97:3700-3706.

Bielmann, V., J. Gillan, N. R. Perkins, A. L. Skidmore, S. Godden, and K. E. Leslie. 2010. An evaluation of Brix refractometry instruments for measurement of colostrum quality in dairy cattle. J. Dairy Sci. 93:3713-3721.

Butler, J. E. 1969. Bovine immunoglobulins-A review. J. Dairy Sci. 52:1895-1909. http://dx.doi.org/10.3168/jds.S00220302(69)86871-2.

Elizondo-Salazar, J. A., and A. J. Heinrichs. 2009. Feeding heat-treated colostrum to neonatal dairy heifers: Effects on growth characteristics and blood parameters. J. Dairy Sci. 92:3265-3273.

Elizondo-Salazar, J. A., B. M. Jayarao, and A. J. Heinrichs. 2010. Effect of heat treatment of bovine colostrum on bacterial counts, viscosity, and immunoglobulin G concentration. J. Dairy Sci. 93:961-967.

Gelsinger, S. L., C. M. Jones, and A. J. Heinrichs. 2015. Effect of colostrum heat treatment and bacterial population on IgG absorption and health of neonatal calves. J. Dairy Sci. http://dx.doi. org/10.3168/jds.2014-8790.

Godden, S. 2008. Colostrum management for dairy calves. Vet. Clin. North Am. Food Anim. Pract. 24:19-39.

Godden, S., S. McMartin, J. Feirtag, J. Stabel, and R. Bey. 2006. Heat-treatment of bovine colostrum. II: Effects of heating dura- tion on pathogen viability and immunoglobulin G. J. Dairy Sci. 89:3476-3483.

Godden, S. M., D. J. Smolenski, M. Donahue, J. M. Oakes, R. Bey, S. Wells, S. Sreevatsan, J. Stabel, and J. R. Fetrow. 2012. Heat-treated colostrum and reduced morbidity in preweaned dairy calves: Results of a randomized trial and examination of mechanisms of effectiveness. J. Dairy Sci. 95:4029-4040.

Johnson, J. L., S. M. Godden, T. Molitor, T. Ames, and D. Hagman. 2007. Effects of feeding heat-treated colostrum on passive transfer of immune and nutritional parameters in neonatal dairy calves. J. Dairy Sci. 90:5189-5198.

McMartin, S., S. Godden, L. Metzger, J. Feirtag, and R. Bey. 2006. Heat treatment of bovine colostrum. I: Effects of temperature on viscosity and immunoglobulin G level. J. Dairy Sci. 89:2110-2118.

Pritchett, L. C., C. C. Gay, D. D. Hancock, and T. E. Besser. 1994. Evaluation of the hydrometer for testing immunoglobulin G1 concentrations in Holstein colostrum. J. Dairy Sci. 77:1761-1767.

Quigley, J. D., A. Lago, C. Chapman, P. Erickson, and J. Polo. 2013. Evaluation of the Brix refractometer to estimate immunoglobulin G concentration in bovine colostrum. J. Dairy Sci. 96:1148-1155.

Tyler, J. W., D. D. Hancock, S. M. Parish, D. E. Rea, and T. E. Besser. 1996. Evaluation of 3 assays for failure of passive transfer in calves. J. Vet. Intern. Med. 10:304-307.

Vetter, A., A. Argueello, C. Baumrucker, and R. M. Bruckmaier. 2013. Short communication: Fractional milking distribution of immunoglobulin G and other constituents in colostrum. J. Dairy Sci. 96:5919-5922.

Weaver, D. M., J. W. Tyler, D. C. VanMetre, D. E. Hostetler, and G. M. Barrington. 2000. Passive transfer of colostral immunoglobulins in calves. J. Vet. Intern. Med. 14:569-577. 